\title{
Biodiversité et écologie de l'avifaune aquatique hivernante dans Garaet Hadj-Tahar (Skikda, Nord-Est de l'Algérie)
}

\author{
Biodiversity and ecology of wintering waterfowl \\ in Garaet Hadj-Tahar (Skikda, North-East Algeria)
}

\author{
S. Metallaoui( ${ }^{(1)}$, M. Houhamdi(2) \\ (1) Département de Biologie, Université du 20 août 1955, Skikda, Algérie \\ metallaoui_s@yahoo.fr \\ (2) Département de Biologie, Université du 08 mai 1945, Guelma, Algérie \\ houhamdimoussa@yahoo.fr
}

Résumé - Dans le but d'inventorier et d'étudier l'écologie de l'avifaune aquatique hivernante dans la Garaet Hadj-Tahar (Nord-Est algérien), des sorties hebdomadaires sont réalisées pendant la saison d'hivernage 2006/2007. Au total 47 espèces appartenant à 15 familles ont été recensées. Les Anatidés (12 espèces et plus de 10000 individus) dont les plus importants sont le Fuligule milouin Aythya ferina (1600 ind.), le Canard chipeau Anas strepera (1560 ind.), le Canard souchet Anas clypeata (2500 ind.) et le Canard siffleur Anas penelope (1200 ind.) ainsi que pour les Rallidés, la Foulque macroule Fulica atra (7100 ind.) sont fortement représentés. Les Anatidés se regroupent dans les endroits dégagés de la Garaet. Les Foulques sont composées de deux populations, l'une autochtone dont les individus se dispersent dans les franges de nénuphars blancs Nymphaea alba et l'autre allochtone, grégaire et plus abondante occupe les régions dégagées du plan d'eau. Les peuplements des Laro-limicoles et des échassiers atteignent leur maximum au début de l'hivernage (750 ind.) et se concentrent sur les berges septentrionales de la Garaet. Cependant, à la fin de la saison d'hivernage, onze espèces dont certaines sont très importantes à l'échelle internationale comme le Fuligule nyroca Aythya nyroca, l'Érismature à tête blanche Oxyura leucocephala et la Poule sultane Porphyrio porphyrio nichent dans la Garaet. L'analyse écologique de ce peuplement nous a montré que la richesse spécifique la plus faible a été enregistrée vers la fin du mois d'août (12 espèces) et la plus élevée pendant la deuxième décade du mois de novembre (32 espèces). Cependant les répartitions entre les espèces les mieux équilibrées sont enregistrées au début de l'hivernage $\left(H^{\prime}=\right.$ $3,299$ et $E=0,744)$. L'analyse statistique multivariée effectuée sur ces dénombrements fournit des informations structurées par une succession temporelle de l'occupation de cette zone humide par l'avifaune aquatique et divise la saison d'hivernage en trois périodes plus ou moins distinctes caractérisées par des regroupements avifaunistiques spécifiques.

Mots clés - Laro-limicoles, Échassiers, Anatidés, hivernage, Garaet, zone humide, conservation

Abstract - In order to identify and study the ecology of the waterfowl wintering in the Garaet Hadj-Tahar (northeastern Algeria) weekly outings are conducted during the winter season of 2006/2007. A total of 47 species belonging to 15 families have been identified. 
The Anatidae (12 species and more than 10000 individuals) the most important being the Common Pochard Aythya ferina (1600 ind.), the Gadwell Anas strepera (1560 ind.), the Northern Sholver Anas clypeata (2500 ind.), the Eurasian Wigeon Anas penelope (1200 ind.) and, among the Rallidae, the Common Coot Fulica atra (7100 ind.) are strongly represented. The Anatidae gather in clear places of the Garaet. The Coots are composed of two populations, one of which is autochthonous and scattered in the fringes of white water lilies Nymphaea alba and the other is allochthonous, gregarious and more abundant and occupies clears areas of the water body. The population of Laro-limicols and Waders reach the maximum at the beginning of the wintering season (750 ind.) and gather together on the northern shores of the Garaet. But at the end of the wintering season, eleven species, some of them are very important at the international level such as Ferruginous Duck Aythya nyroca, White-headed Duck Oxyura leucocephala and Purple Swamphen Porphyrio porphyrio nest in the Garaet. The environmental analysis of this population has shown that the lowest the species richness was recorded toward the end of August (12 species) and the highest one during the second ten days of November (32 species). But the best balanced interspecies distribution is recorded at the beginning of the wintering season $\left(H^{\prime}=3.299\right.$ and $E=0.744)$. The statistical multivariate analysis performed on these counts outlines a structure following a temporal sequence in the occupation of Garaet by aquatic birds and divides the wintering season in three more or less distinct periods characterized by specific clusters of the waterfowl.

Key words - waders, shorebirds, Anatidae, wintering, Garaet, wetland, conservation

\section{INTRODUCTION}

La Numidie située dans le Nord-Est algérien, est réputée pour ses zones humides qui sont réparties en deux grands complexes séparés par l'Oued Seybouse : la Numidie orientale composée des complexes de Annaba et d'El-Kala et la Numidie occidentale représentée par le complexe des zones humides de Guerbes-Sanhadja (Samraoui \& De Bélair, 1997). La Numidie orientale a pour limite septentrionale la méditerranée et les frontières algéro-tunisiennes la délimitent à l'Est. Cette région de l'Algérie renferme un grand nombre de sites humides exceptionnels de par leurs dimensions et leurs diversités et où plusieurs études scientifiques ont été menées (Ledant \& Van Dijk, 1977; Van Dijk \& Ledant, 1980; Morgan, 1982; Samraoui et al., 1992; Boumezbeur, 1993; Samraoui \& De Belair, 1998;
Chalabi, 1998; Houhamdi, 1998). Le complexe de Guerbes-Sanhadja est une plaine homogène de 42100 ha. Cependant il n'a bénéficié pratiquement d'aucune étude scientifique mis à part celle menée par Samraoui et De Bélair qui a signalé la valeur particulière de ce complexe pour le maintien de la biodiversité (Samraoui \& De Belair, 1994). Par contre, dans les zones humides de la Numidie orientale qui occupent une superficie de $17500 \mathrm{~h}$, des comptages hivernaux réguliers ont souligné le rôle majeur joué par le complexe d'El Kala, pour l'hivernage des Anatidés avec une moyenne estimée de 90000-100000 individus (Ledant et al., 1981; Isenmann \& Moali, 2000). Des travaux reposant sur les recensements et les inventaires des effectifs fréquentant nos écosystèmes aquatiques et sur l'étude de leur écologie "dynamique des populations, étude du 

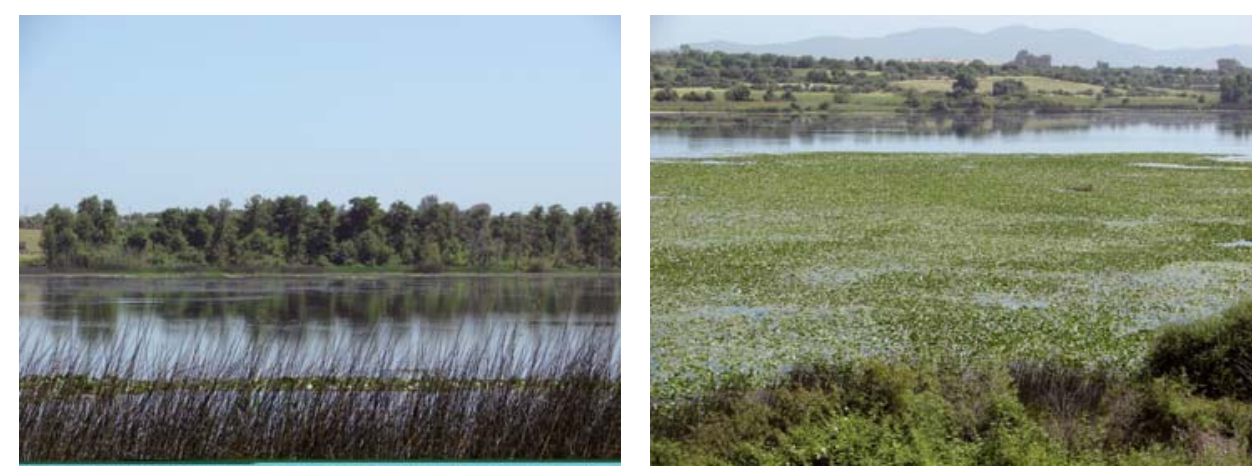

Photos 1 et 2. Photos de Garaet Hadj-Tahar prises le 7 avril 2007 (par Houhamdi M.).

Photos 1 et 2. Garaet Hadj Tahar photos taken on April 7th 2007 (by Houhamdi M.).

comportement diurne et nocturne, écologie et biologie de la reproduction des espèces nicheuses... » ont également été effectués par Samraoui et al. (1992), Samraoui \& De Belair (1997, 1998); Rizi et al. (1999); Houhamdi \& Samraoui $(2001,2002,2003$, 2008), Samraoui \& Houhamdi (2001), Samraoui et al. (2006), Boulekhssaim et al. 2006a, 2006b, Samraoui \& Samraoui (2007). Notre travail a pour objectif de mettre en exergue l'importance et le rôle écologique de Garaet Hadj-Tahar (complexe des zones humides de Guerbes-Sanhadja, Nord-Est algérien) pour l'hivernage des oiseaux d'eau et repose dans un premier lieu sur le recensement de toutes les espèces avifaunistiques aquatiques ayant fréquenté et utilisé ce plan d'eau, puis dans un second rang la détermination de leurs phénologies et l'étude de leur écologie.

\section{MATÉRIEL ET MÉTHODES}

\section{Description du site}

Garaet Hadj-Tahar est un étang d'eau douce de 100 ha (photos 1 et 2) situé dans la commune de Ben-Azouz, entre la latitude $36^{\circ} 51^{\prime} 50^{\prime \prime} \mathrm{N}, \quad 07^{\circ} 15^{\prime} 57^{\prime \prime} \mathrm{E}$ et à une altitude de $16 \mathrm{~m}$. II est site Ramsar depuis le 2 février 2001 et fait partie du complexe des zones humides de Guerbes-Sanhadja formé de lagunes salées comme Garaet Dahria, de lacs d'eau douce tel que Garaet Sidi Makhlouf et de marais d'eau douce. Administrativement, il appartient à la wilaya de Skikda (Numidie occidentale, Nord-Est algérien) bordée à l'ouest par les collines côtières de Skikda et à l'Est par le massif côtier de Chetaibi. Les altitudes de la zone se situent entre 0 et $200 \mathrm{~m}, 48,5 \%$ des terres ont une pente inférieure ou égale à $3 \%$ et le reste à 12,5\% (Boumezbeur, 2001).

Ce plan d'eau douce situé à une vingtaine de kilomètres de la Méditerranée présente une forme ovale très allongée (Fig. 1) et joue un rôle important pour l'avifaune aquatique, de nombreuses espèces l'utilisant pendant toute l'année (Metallaoui \& Houhamdi, 2008). II exhibe aussi une diversité floristique très abondante à espèces rares, où l'on rencontre Nymphaea alba, Typha angustipholia, Phragmites 


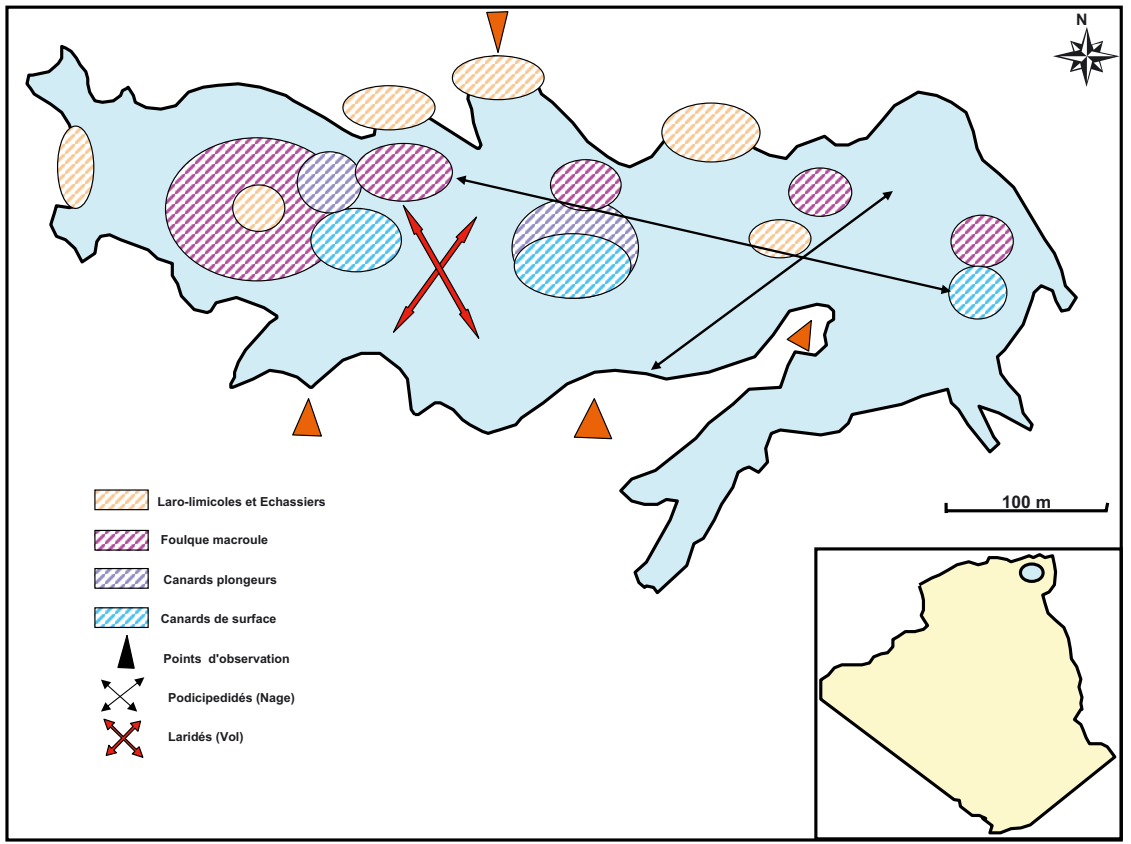

Fig. 1. Situation géographique de Garaet Hadj-Tahar (Skikda, Algérie) et occupation spatiale de l'avifaune aquatique.

Fig. 1. Geographical situation of Garaet Hadj-Tahar (Skikda, Algeria) and waterfowl spatial occupation.

australis, Scirpus maritimus, S. lacustris, Iris pseudoacaurus occupant pratiquement 60 à $70 \%$ de la superficie totale du plan d'eau, ainsi qu'une ptéridophyte Salvinia natans. La Garaet est bordée d'une ceinture de végétation composée principalement de Juncus acutus, J. maritimus, Olea europea, Asphodelus aestivus, Rubus ulmifolius et de pelouses de graminées dont les plus abondantes sont Cynodan dactylon et Paspalum distichum. Les terres entourant le site sont exclusivement utilisées par les riverains pour la culture maraîchère notamment la pastèque, le melon, la tomate... La profondeur moyenne de l'eau varie entre 0,8 et $1,20 \mathrm{~m}$. Elle augmente subitement suite aux chutes torren- tielles de pluies, du fait que la Garaet constitue en réalité une cuvette qui reçoit continuellement les ruissellements des eaux de pluies des montagnes environnantes.

\section{Méthodologie}

Des sorties hebdomadaires étaient réalisées pratiquement de $8 \mathrm{~h}$ à $16 \mathrm{~h}$ depuis le mois d'août 2006 jusqu'au mois d'avril 2007. Les dénombrements et les recensements des populations aviennes, ont été réalisés depuis plusieurs points d'observation (Fig. 1) à l'aide d'une longue vue KONUS $20 \times 65$. Nous avons essayé de réaliser des comptages individuels des 
oiseaux d'eau si le groupe ou la population compte moins de 200 individus et se trouve à une distance proche n'excédant pas les $200 \mathrm{~m}$. Dans le cas contraire, si le groupe ou la population d'oiseaux d'eau est éloigné et compte un effectif assez important, nous procédons à des estimations visuelles (Blondel, 1975; Tamisier \& Dehorter, 1999). Cette méthode est la plus employée dans les recensements hivernaux de l'avifaune aquatique et présente cependant une marge d'erreur variant entre 5 et $10 \%$ dépendant de l'expérience de l'observateur, du matériel utilisé et de la structure du milieu : ceinture de la végétation et hauteur des hélophytes (Lamotte \& Bourlière, 1969 ; Houhamdi, 2002).

\section{RÉSULTATS ET DISCUSSIONS}

\section{Phénologie et évolution de l'abondance de l'avifaune aquatique}

Tout au début de la saison d'hivernage (mois d'août), le peuplement avifaunistique est particulier car la période de reproduction n'est pas encore terminée et tous les oiseaux présents n'ont pas encore adopté le grégarisme typique de la période hivernale. Le graphique de l'évolution de l'abondance des effectifs d'oiseaux d'eau dans Garaet Hadj-Tahar divise la saison d'hivernage en trois périodes (Fig. 2). La première s'étale d'août à décembre où le peuplement total fluctue entre 1000 et 4000 individus dominés principalement par les Anatidés (3000 individus) regroupant les espèces sédentaires avec un faible effectif présentées par le canard colvert Anas platyrhynchos (5-1000 individus), le Fuligule nyroca Aythya nyroca (30-800 individus), l'Érismature à tête blanche Oxyura leucocephala (4-81 individus), le Fuligule milouin (5-800 individus). Les hivernants les plus précoces sont représentés le Canard souchet Anas clypeata (600 individus) et la Sarcelle d'hiver Anas crecca crecca (800 individus).

La deuxième période commence au mois de janvier et se poursuit jusqu'à la fin mars; elle se caractérise par une augmentation brusque de l'effectif total où il atteint un maximum de 12000 individus le 28 janvier 2007. L'abondance des Rallidés a triplé par l'arrivée de la population allochtone de la Foulque macroule Fulica atra (Fig. 2) occupant la partie Est du plan d'eau (Fig. 1) et manifeste un comportement grégaire d'où l'importance des effectifs accusés au mois de février (7100 individus). On note également l'arrivée de populations de passage prénuptial d'Anatidés des autres quartiers d'hivernage. Nous observons ainsi le Fuligule milouin $A y$ thya ferina (1600 individus), le Canard chipeau Anas strepera (1500 individus), le Canard souchet Anas clypeata (2500 individus) et le Canard siffleur Anas penelope (1200 individus). Ils se regroupent dans des endroits égagés du plan d'eau (Fig. 1).

La troisième période débute dès le mois de mars et caractérise la fin de la saison d'hivernage. Nous assistons à un effondrement de l'effectif total de toutes les espèces traduit par la migration prénuptiale vers leurs lieux habituels de reproduction. Les peuplements des Laro-Limicoles et Échassiers composés de 30 espèces appartenant à 12 familles (Tab. I) sont 


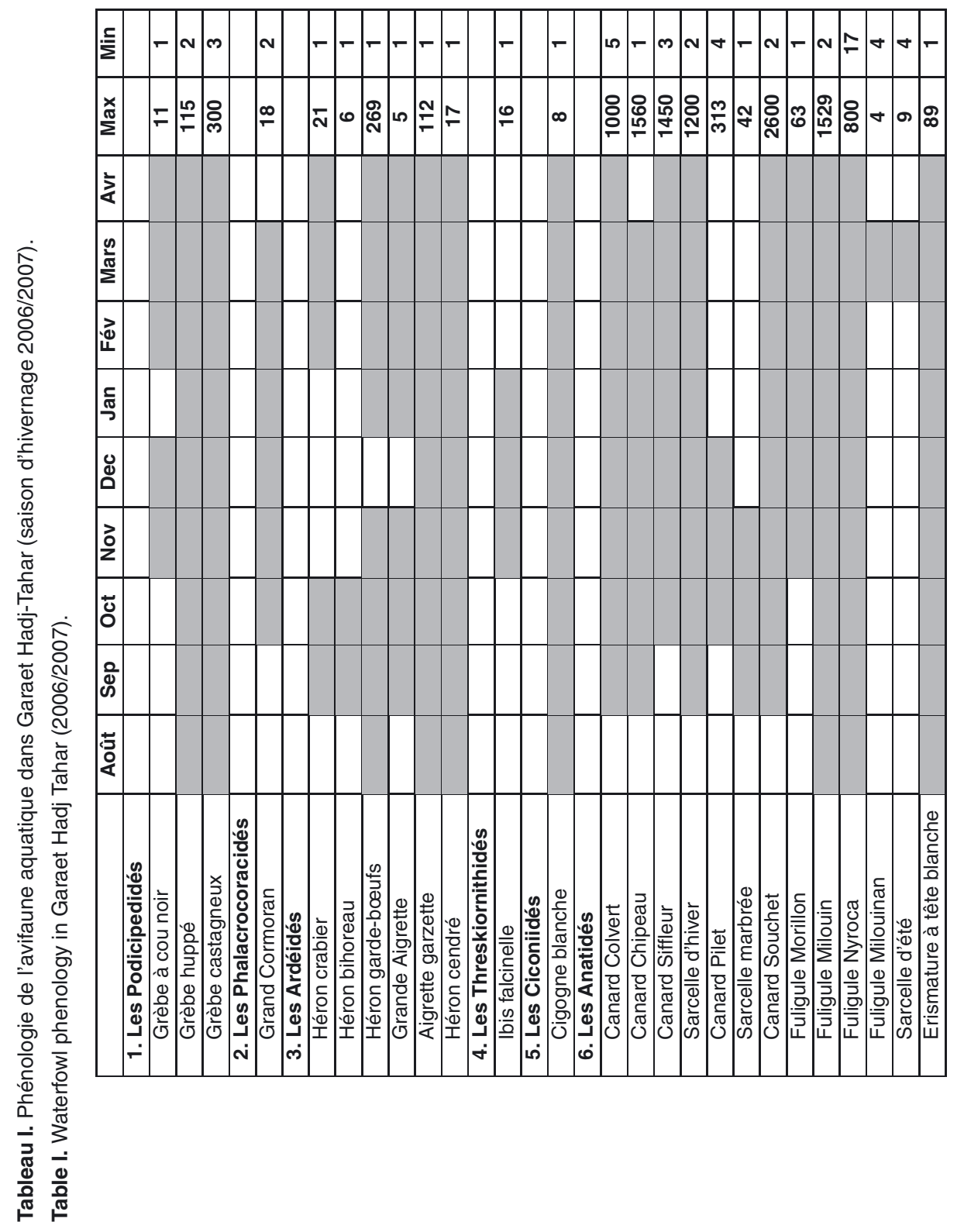




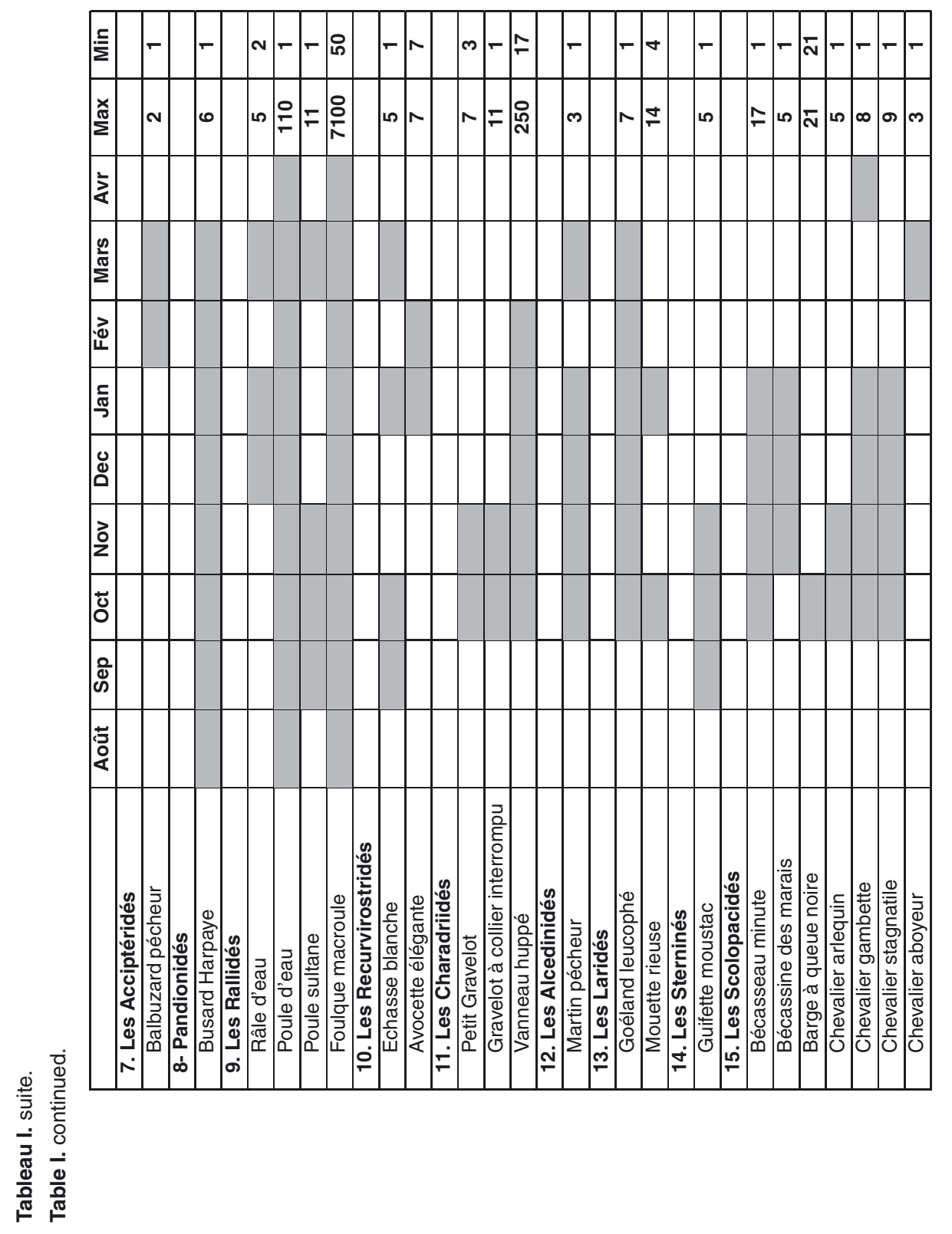



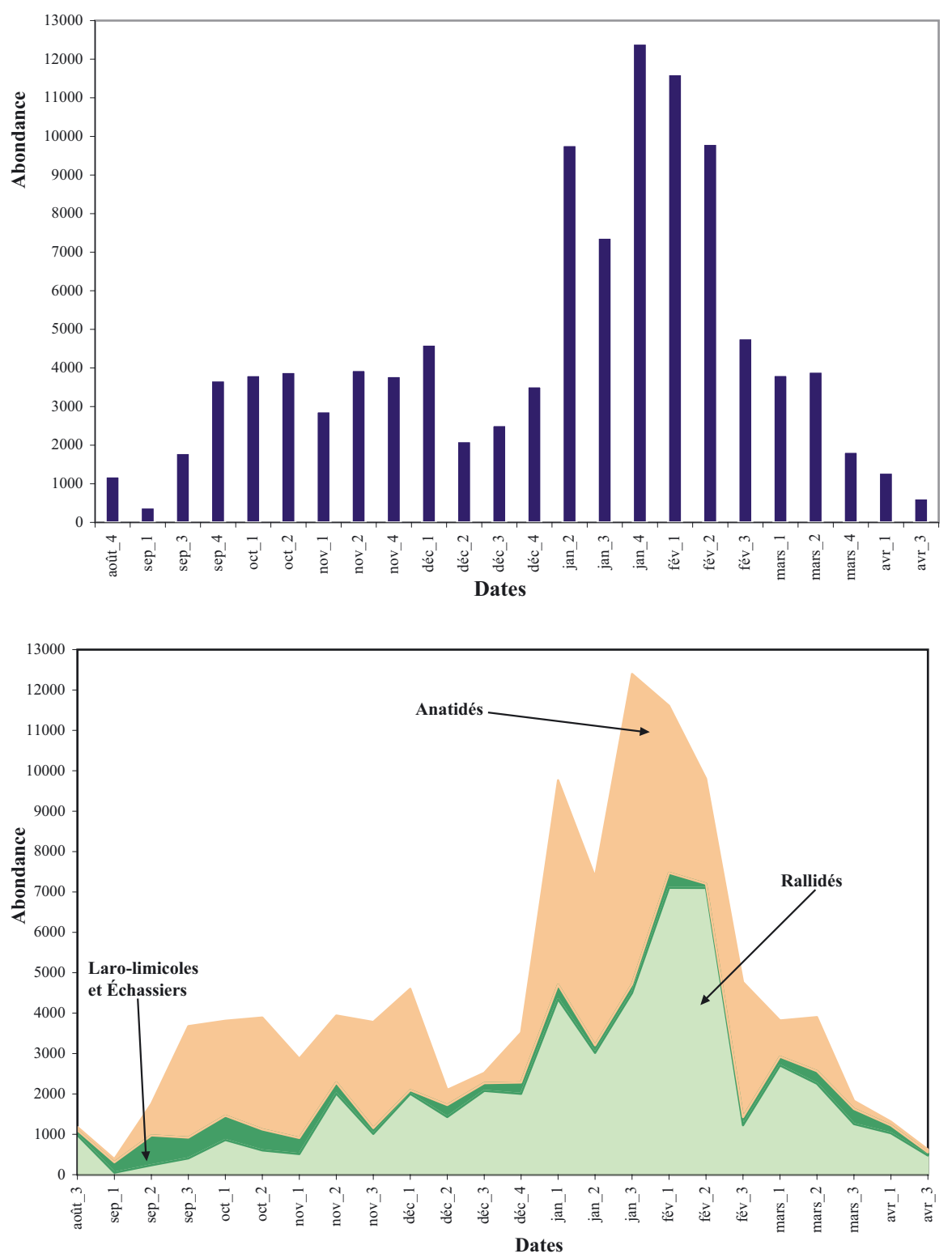

Fig. 2. Évolution de l'abondance de l'avifaune aquatique d'août 2006 à avril 2007.

Fig. 2. Evolution of waterfowl abundance from August 2006 to April 2007. 
faiblement représentés dans le site. Le maximum enregistré a été observé durant le début de la saison d'hivernage : 739 individus (Fig. 2). Ainsi, ces oiseaux ont surtout occupé la Garaet durant les périodes de basses eaux rendant l'accès à leur nourriture facile. Lorsque le niveau d'eau augmente nous observons les canards plongeurs et de surface, les Grands Cormoran Phalacrocorax carbo venus de la Méditerranée pour s'alimenter d'anguille Anguilla anguilla et quelques Goélands leucophée Larus michahellis entretenant leurs plumages en le rinçant du sel marin.

D'une manière générale, le graphique de l'abondance affiche un faible effectif (386-4000 individus) entre le début de l'étude et les premières semaines d'octobre (Fig. 2). Mais à partir de sa dernière décade, l'effectif augmente par l'arrivée des hivernants précoces composés principalement d'Anatidés comme le Canard colvert, le Canard souchet, la Sarcelle marbrée, la Sarcelle d'hiver... Cet effectif reste plus ou moins stable et ce jusqu'à janvier. Au-delà de cette date, nous notons une élévation très rapide de l'effectif le rapportant de 9775 à 12404 individus (maximum comptabilisé durant toute l'étude). Ces regroupements, sont révélateurs de la préparation à la migration prénuptiale traduite par un effondrement progressif de l'effectif total. À la fin de l'étude, il ne reste dans la Garaet que les nicheurs sédentaires (Anatidés, Foulques et Grèbes) ainsi que les estivants nicheurs comme la Cigogne blanche Ciconia ciconia. Ainsi, durant nos dénombrements, cet étang s'est montré un lieu propice pour la survie de nombreux peuplements d'oiseaux d'eau dont les effectifs sont considérablement variés avec une représentativité qualitative et quantitative des Anatidés et des Foulques macroules.

\section{Évolution des paramètres écologiques}

\section{Richesse spécifique}

Le graphique de la richesse spécifique quant à lui montre une allure en $(m)$ exhibant deux pics l'un pendant les deux premières semaines du mois de novembre avec 32 espèces et le second à la fin de la deuxième décade de janvier avec 29 espèces (Fig. 3). Le minimum est observé à la fin août avec 12 espèces. La richesse spécifique augmente progressivement avec l'arrivée des hivernants (le Canard souchet, la Sarcelle d'hiver, le Canard pilet et le Canard chipeau...) et par le passage de certaines espèces : le Héron bihoreau Nycticorax nycticorax, le Héron crabier Ardeola ralloides, l'Échasse blanche Himantopus himantopus... pour atteindre son maximum au mois de novembre. Audelà de janvier, on note une réduction de la richesse spécifique traduite par la dispersion de quelques espèces la Sarcelle marbrée, la Grande aigrette Ardea alba, le Petit gravelot Charadrius dubius... La faible représentativité spécifique enregistrée pendant le mois de décembre est principalement due aux conditions climatiques (des vents violents et des pluies torrentielles) induisant une visibilité limitée. Après cette date, nous remarquons une deuxième élévation expliquée par le passage des espèces suivantes la 


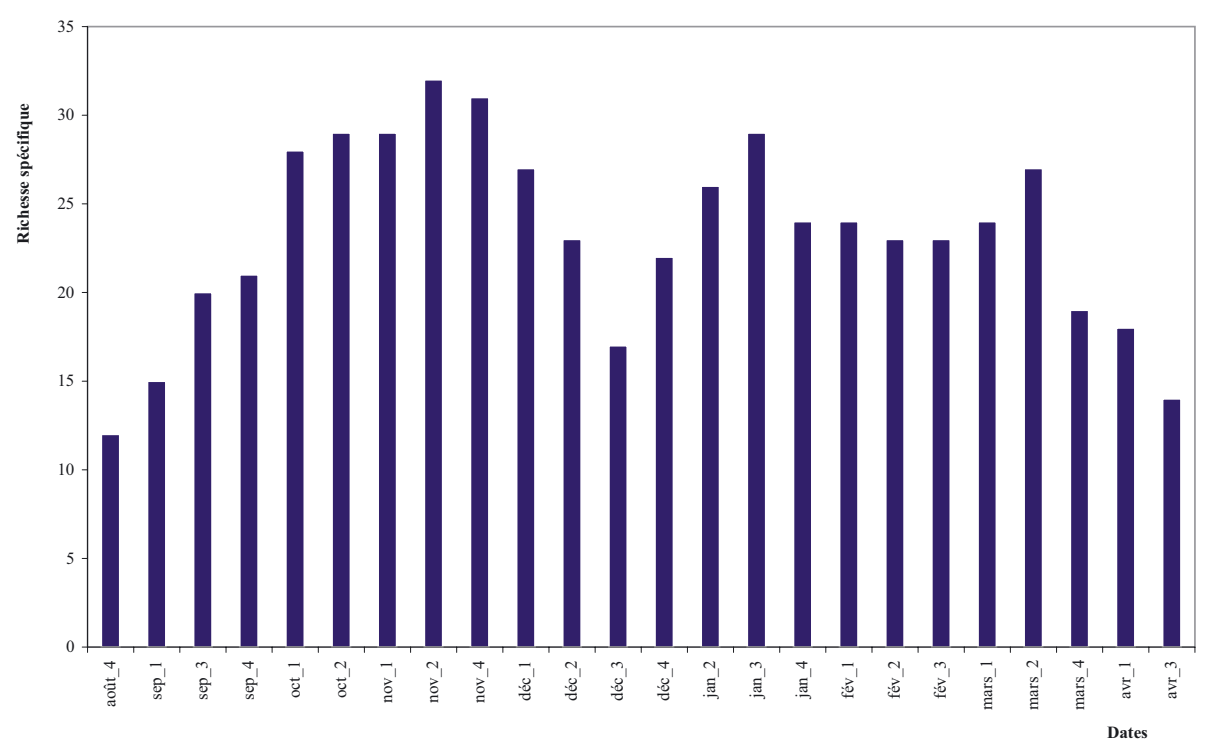

Fig. 3. Évolution de la richesse spécifique de l'avifaune aquatique d'août 2006 à avril 2007.

Fig. 3. Evolution of waterfowl specific richness from August 2006 to April 2007.

Grande aigrette, l'Ibis falcinelle Plegadis falcinellus, la Mouette rieuse Larus ridibundus, l'Avocette élégante Recurvirostra avosetta... ramenant la richesse totale à 29 espèces au-delà de la mi-janvier, puis la richesse indique une stabilité et ce jusqu'à la mi-mars où nous assistons à une légère élévation due à l'observation d'espèces erratiques telles qu'une Poule sultane, quatre Sarcelle d'été Marmaronetta querquedula, deux Martins pêcheurs Alcedo atthis, un Chevalier guignette Actitis hypoleucos et un Petit gravelot Charadrius dubius suivie d'un effondrement progressif.

Globalement, Garaet Hadj-Tahar a hébergé durant la saison d'hivernage (2006-2007) 47 espèces d'oiseaux d'eau (Tab. I) avec un maximum de 32 espèces observées à la deuxième dé- cade du mois de novembre et un minimum de 12 espèces la fin août (Fig. 3).

\section{Indices de diversité}

Les graphiques des indices écologiques étudiant l'équilibre des populations (indice de diversité de Shannon $H^{\prime}$ et indice d'équitabilité $E$ ) montrent des allures plus ou moins similaires (Figs. 4 et 5). D'une manière générale les maximums sont observés au début de l'hivernage soit pendant les mois de septembre, octobre et novembre. Pendant cette période la Garaet est à son niveau d'eau le plus faible. Le site est occupé par un faible effectif mais d'une grande représentativité spécifique composé principalement des hivernants. Les valeurs les plus élevées pour les deux indices sont observées 


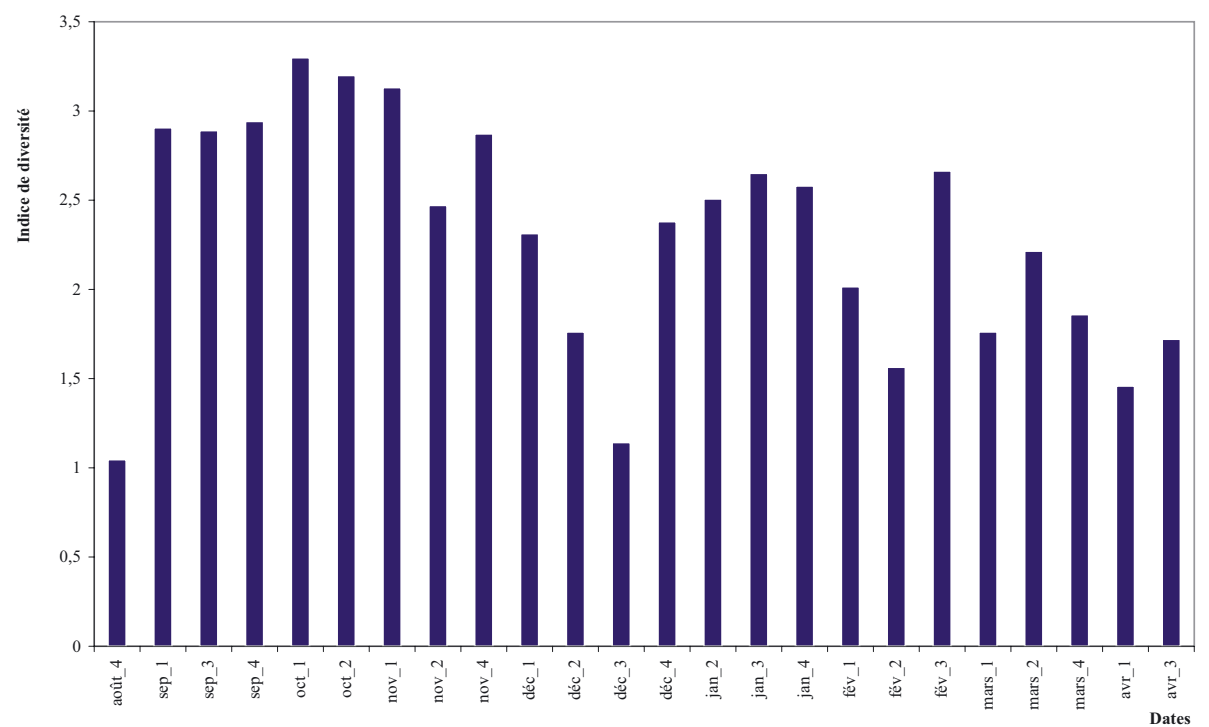

Fig. 4. Évolution de l'indice de diversité de Shannon de l'avifaune aquatique d'août 2006 à avril 2007. Fig. 4. Evolution of waterfowl diversity index from August 2006 to April 2007.

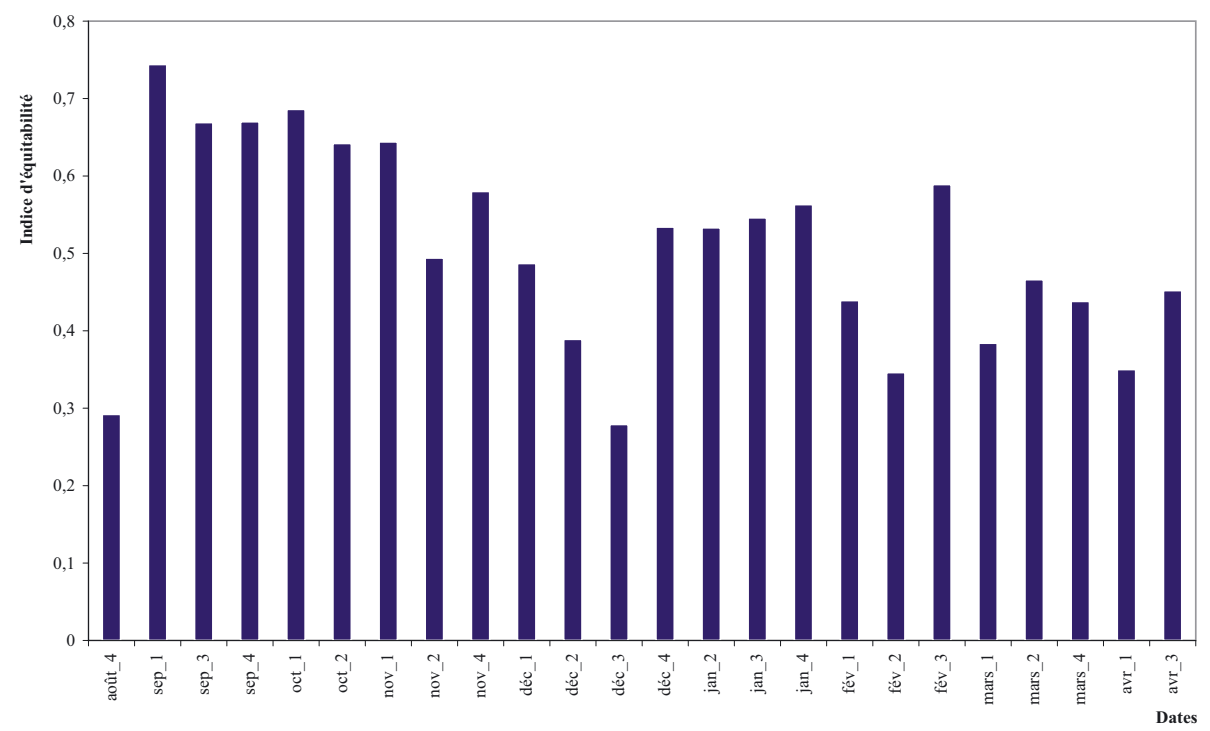

Fig. 5. Évolution de l'indice d'équitabilité de l'avifaune aquatique d'août 2006 à avril 2007.

Fig. 5. Evolution of waterfowl equitability index from August 2006 to April 2007. 
après cette période. Le maximum noté pour l'indice de diversité de Shannon est de 3,5 lors de la première décade du mois d'octobre (Fig. 4) et de 0,73 pour l'indice d'équitabilité pendant la première semaine de septembre (Fig. 5). Après cette période, le niveau d'eau a augmenté et nous observons des variations en dents de scie provoquées par le départ de certaines espèces et à l'observation d'autres plus rares dans la garaet comme le Petit gravelot, le Fuligule milouinan Aythya marila (Metallaoui \& Houhamdi, 2007) et la Sarcelle d'été Anas querquedula mais surtout une domination des peuplements aviens représentée par la Foulque macroule (7100 individus), le Canard souchet (2600 individus), le Fuligule milouin (1529 individus), le Canard siffleur (1450 individus) et le Canard chipeau (1560 individus).

\section{Analyse statistique multivariée des données}

L'analyse statistique multivariée effectuée sur les dénombrements hebdomadaires de la saison d'hivernage (2006-2007) et exprimée sur le plan factoriel $1 \times 2$ de l'AFC (Analyse Factorielle des Correspondances) qui détient $52 \%$ de l'inertie nous expose d'une part des informations structurées par une succession temporelle de l'occupation de la Garaet par l'avifaune aquatique, et d'autre part, et sous un autre angle l'axe des ordonnées oppose le début de l'hivernage, à l'hivernage proprement dit et à sa fin (Fig. 6). L'axe des abscisses distingue principalement les mois de novembre et décembre par rapport aux autres périodes. Ainsi, le graphique montre que la saison d'hivernage est en réalité formée de trois périodes plus ou moins distinctes correspondants aux trois groupements avifaunistiques.

- Le premier groupement correspond au début d'hivernage qui s'étale entre la fin août et le mois d'octobre et regroupe principalement les nicheurs sédentaires comme le Fuligule nyroca, l'Érismature à tête blanche, le Grèbe huppé, le Grèbe castagneux, le Canard colvert, la Poule d'eau et la Poule sultane. Ce premier groupe inclus également des populations de passage précoce comme la Sarcelle marbrée, ainsi que le Chevalier gambette Tringa totanus et le Chevalier arlequin Tringa erythropus, la Barge à queue noire Limosa limosa, l'Échasse blanche, le Héron crabier, le Héron bihoreau et le Héron cendré Ardea cinerea et des groupements post-nuptiaux de Hérons Garde bœufs et d'aigrettes garzette nichant dans la Numidie (Belhadj et al., 2007; Samraoui et al., 2007).

- Le second groupe correspond à la période d'hivernage notée durant les mois de novembre et décembre fortement représenté d'hivernants retardataires tels que certains Anatidés comme le Canard pilet, le Fuligule milouin, le Fuligule morillon, le Canard chipeau et le Canard souchet. Ce second groupe comprend également des populations de passage marquées par le Vanneau huppé Vanellus vanellus, l'lbis falcinelle, le Grand cormoran, le Chevalier guignette Actitis hypoleucos, le Chevalier stagnatile Tringa stagnatilis, la Bécassine des 

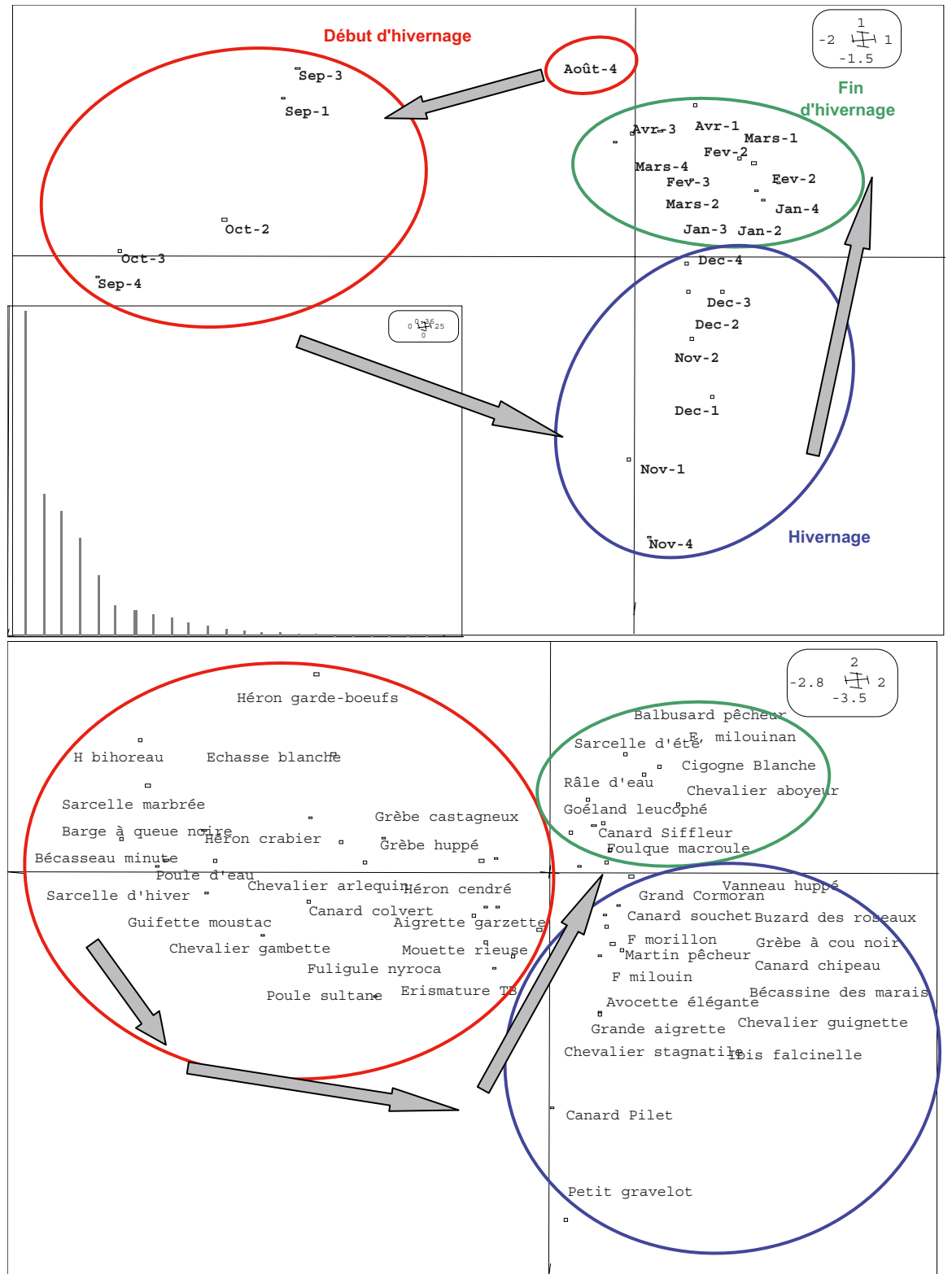

Fig. 6. Plan factoriel $1 \times 2$ de l'analyse factorielle des correspondances des dénombrements de l'avifaune aquatique de Garaet Hadj-Tahar (24 sorties $\times 47$ espèces). Axes d'inertie : 0,36, 0,16, 0,13, $0,11,0,07$ et 0,03 .

Fig. 6. Factorial map $1 \times 2$ of correspondences analysis of waterfowl abundance of Garaet Hadj Tahar. Inertia axes: $0.36,0.16,0.13,0.11,0.07$ and 0.03 . 
marais Gallinago gallinago, l'Avocette élégante et le Martin pêcheur, ainsi que des visiteurs accidentels comme le Petit gravelot.

- La troisième période, plus longue allant de janvier à avril et rassemblant un nombre élevé d'espèces est caractérisée par des rassemblements pré-migratoires de canards siffleurs et par la population allochtone de foulque macroule. Cette période correspond également à la fréquentation du site par des espèces nicheuses dans la région et sur le littoral méditerranéen comme le Goéland leucophée (Moulai, 2007; Moulai et al., 2005, 2006), la Cigogne blanche, le Râle d'eau et le Balbuzard pêcheur Pandion haliaetus. Nous avons observé aussi des espèces de passage comme la Sarcelle d'été et le Chevalier aboyeur Tringa nebularia.

\section{CONCLUSION}

La saison d'hivernage des oiseaux d'eau de Garaet de Hadj-Tahar est divisée en trois périodes. Le début de l'hivernage est caractérisé par des effectifs faibles, une richesse spécifique importante, les meilleurs équilibres et un niveau d'eau faible. L'hivernage proprement dit est caractérisé par un niveau d'eau élevé dû aux chutes de pluies hivernales. L'effectif total demeure faible; cependant une richesse spécifique élevée traduit une domination de certaines espèces notamment les populations d'Anatidés hivernants. La fin de l'hivernage est caractérisée par des regroupements prémigratoires et des départs progressifs des espèces vers leurs lieux de nidification habituels. Garaet Hadj-Tahar joue cependant un rôle important pour l'avifaune aquatique nicheuse, particulièrement les populations d'Anatidés, de Rallidés et de Podicipedidés et aux oiseaux de passage.

Malgré son statut, la Garaet se heurte à des problèmes de conservation où l'on constate une extension anarchique de l'agriculture (culture très prisée dans la région du melon et de la pastèque) qui nécessite un pompage d'eau intensif. En outre, le braconnage et la chasse non réglementée influencent l'hivernage et la nidification de ces peuplements aviens.

\section{REMERCIEMENTS}

Ce travail s'inscrit dans le cadre d'une préparation de thèse de doctorat en écologie et environnement par le premier auteur et par son biais nous voulons remercier toutes les personnes qui ont contribué à sa réalisation, et plus particulièrement M. Gasmi Abdelatif, Conservateur des forêts de la wilaya de Batna (Algérie) qui a véritablement soutenu ce travail et la famille Litim (commune de Ben-Azouz). Nos remerciements vont également aux deux référés qui ont accepté de corriger cet article.

\section{RÉFÉRENCES BIBLIOGRAPHIQUES}

Belhadj G., Chalabi B., Chabi Y., Kayser Y. \& Gauthier-Clerc M. 2007. Le retour de l'lbis falcinelle Plegadis falcinellus nicheur en Algérie. Aves 44 (1) : 29-36.

Blondel J., 1975. Analyse des peuplements d'oiseaux d'eau. Elément d'un diagnostic écologique I : La méthode des échantillonnage fréquentiels progressifs (E.F.P.). Terre et Vie 39 : 533589. 
Boulekhssaim M., Houhamdi M. \& Samraoui B., 2006a. Status and diurnal behaviour of the Shelduck Tadorna tadorna in the Hauts Plateaux, Northeast Algeria. Wildfowl 56 : 65-78.

Boulekhssaim M., Houhamdi M., Saheb M., Samraoui-Chenafi F. \& Samraoui B., 2006b. Breeding and banding of Greater flamingo Phoenicopterus roseus In Algeria, August 2006. Flamingo $14:$ : 21-24.

Boumezbeur A., 1993. Ecologie et biologie de la reproduction de l'Erismature à tête blanche Oxyura leucocephala et du Fuligule nyroca Fuligula nyroca sur le Lac Tonga et le Lac des Oiseaux (NordEst algérien). Thèse de doctorat, USTL. Montpellier, $250 \mathrm{pp}$.

Boumezbeur A., 2001. Atlas des zones humides algériennes d'importance internationales. Direction Générale des Forêts, Ben Aknoun Alger, 65 pp.

Chalabi B., 1998. Contribution à l'étude de l'importance des zones humides algériennes pour la protection de l'avifaune: cas du Lac Tonga (P.N.E.K.). Thèse de magister. INA, Alger, 133 pp.

Houhamdi M., 1998. Ecologie du Lac des Oiseaux : cartographie, palynothèque et utilisation de l'espace par l'avifaune aquatique. Thèse de magister, Univ. Annaba, $198 \mathrm{pp}$.

Houhamdi M., 2002. Ecologie des peuplements aviens du Lac des Oiseaux (Numidie orientale). Thèse de doctorat d'état, Univ. Annaba, 238 pp.

Houhamdi M. \& Samraoui B., 2001. Diurnal time budget of wintering Teal Anas crecca crecca L. at Lac des Oiseaux, northeast Algeria. Wildfowl 52 : 87-96.

Houhamdi M. \& Samraoui B., 2002. Occupation spatio-temporelle par l'avifaune aquatique du Lac des Oiseaux (Algérie). Alauda 70 : 301310.

Houhamdi M. \& Samraoui B., 2003. Diurnal behaviour of wintering Wigeon Anas penelope. in Lac des Oiseaux, northeast Algeria. Wildfowl. 54 : 51-62.

Houhamdi M. \& Samraoui B., 2008. Diurnal and nocturnal behaviour of Ferruginous Duck Aythya nyroca at
Lac des Oiseaux, northeast Algeria. Ardeola.

Isenmann P. \& Moali A., 2000. Oiseaux d'Algérie/Birds of Algeria. Paris: Société d'Etudes Ornithologiques de France, Muséum National d'Histoire Naturelle. 336 p.

Lamotte J. \& Bourlière A., 1969. Problèmes d'écologie: l'échantillonnage des peuplements animaux des milieux terrestres. Masson, $218 \mathrm{pp}$.

Ledant J.P. \& Van Dijk G., 1977. Situation des zones humides algériennes et leur avifaune. Aves 14 : 49-53.

Ledant J.P., Jacob P., Jacobs F., Malher B., Ochando J. \& Roché J., 1981. Mise à jour de l'avifaune algérienne. $L e$ Gerfaut 71 : 295-398.

Metallaoui S. \& Houhamdi M., 2007. Une observation du Fuligule milouinan Aythya marila en Algérie. Alauda. 75 (3) : 77.

Metallaoui S. \& Houhamdi M., 2008. Données préliminaires sur l'avifaune aquatique de la Garaet Hadj-Tahar (Skikda, Nord-Est algérien). ABC 15 (1) : 71-76.

Morgan N.C., 1982. An ecological survey of standing waters in North-West Africa : II Site descriptions for Tunisia and Algeria. Biol. Cons 24 : 83-113.

Moulai R., 2007. Fluctuation saisonnière des oiseaux de la décharge publique de la ville de Béjaia (Algérie), cas particulier du Goéland leucophée Larus michahellis. Ostrich 78 (2) : 527-531.

Moulai R., Saadoul N. \& Doumandji S., 2005. Nidification urbaine et à l'intérieur des terres du goéland leucophée Larus michahellis. En Algérie. Alauda 73 (3) : 195-200.

Moulai R., Saadoul N. \& Doumandji S., 2006. Effectifs et biologie de la reproduction du goéland leucophée Larus michahellis dans la région de Bejaia. Alauda 74 (2) : 225-234.

Rizi H., Benyacoub S., Chabi Y. \& Baňbura J., 1999. Nesting and reproduction characteristics of coots Fulica atra breeding on two lakes in Algeria. Ardeola 46 : 179-186 
Samraoui B. \& De Belair G., 1994. Death of a lake : Lac noir in Northeastern Algeria. Environmental Conservation. 21 (2) : 169-172.

Samraoui B. \& De Belair G., 1997. The Guerbes-Sanhadja wetlands : Part I, Overview. Ecology 28 : 233-250.

Samraoui B. \& De Belair G., 1998. Les zones humides de la Numidie orientale: Bilan des connaissances et perspectives de gestion. Synthèse (Numéro spécial) $4: 90 \mathrm{pp}$.

Samraoui B. \& Houhamdi M., 2001. Première observation de l'Erismature rousse Oxyura jamaiensis en Algérie. Alauda 63 : 396.

Samraoui F. \& Samraoui B., 2007. The Reproductive Ecology of the Common coot Fulica atra in the Hauts Plateaux; Northeast Algeria. Waterbirds 30 (1) : 133-139

Samraoui B., De Belair G. \& Benyacoub S., 1992. A much threatened lake : Lac des Oiseaux (North-east Algeria).
Environmental Conservation 19 : 264267 et 276.

Samraoui B., Ouldjaoui A., Boulakhssaim M., Houhamdi M., Saheb M. \& Bechet A., 2006. The first recorded reproduction of the Greater Flamingo Phoenicopterus roseus in Algeria: behavioural and ecological aspects. Ostrich 77 (3\&4) : 153-159.

Samraoui F., Menai R. \& Samraoui B., 2007. Reproductive ecology of the Cattle Egret Bubulcus ibis at Sidi Achour, North-eastern Algeria. Ostrich 78 (2) : 481-487.

Tamisier A. \& Dehorter O., 1999. Camargue, Canards et Foulques: Fonctionnement d'un prestigieux quartier d'hiver. Nîmes : Centre Ornithologique du Gard. 369 pp.

Van Dijk G. \& Ledant J.P., 1980. Rapport d'observation sur les oiseaux dans la région d'Annaba. Rapport dactylographié, $8 \mathrm{pp}$. 